\title{
Retrievals of Jovian tropospheric phosphine from Cassini/CIRS
}

\author{
P.G. J. Irwin ${ }^{1}$, P. Parrish ${ }^{1}$, T. Fouchet ${ }^{1}$, S.B Calcutt ${ }^{1}$, F.W. Taylor ${ }^{1}$, A.A. Simon-Miller ${ }^{2}$, \\ and C.A. Nixon ${ }^{3}$ \\ ${ }^{1}$ Atmospheric, Oceanic, and Planetary Physics, University of Oxford, Clarendon \\ Laboratory, Parks Rd, Oxford, OX1 3PU, United Kingdom. \\ Tel: (+44) 1865 272933, Fax: (+44) 1865272923. \\ E-mail: irwin@atm.ox.ac.uk \\ ${ }^{2}$ NASA Goddard Space Flight Center, Greenbelt, MD 20771, U.S.A. \\ ${ }^{3}$ University of Maryland, College Park, MD 20742, U.S.A.
}

Submitted to Icarus

Number of manuscript pages: $23+$ acknowledgements, references, tables and figures (39 in total).

Number of Tables: 2

Number of Figures: 11 
Proposed running head:

CIRS Phosphine retrievals

Editorial correspondence should be directed to:

Dr Patrick G. J. Irwin,

Atmospheric, Oceanic and Planetary Physics, Clarendon Laboratory, Parks Rd, Oxford OX1 3PU, United Kingdom.

Telephone: (+44) $1865272933 \quad$ (272083 for direct line)

Fax: $\quad$ (+44) 1865272923

Email: $\quad$ irwin@atm.ox.ac.uk 


\begin{abstract}
On December $30^{\text {th }} 2000$, the Cassini-Huygens spacecraft reached the perijove milestone on its continuing journey to the Saturnian system. During an extended sixmonth encounter, the Composite Infrared Spectrometer (CIRS) returned spectra of the Jovian atmosphere, rings and satellites from $10-1400 \mathrm{~cm}^{-1}(1000-7 \mu \mathrm{m})$ at a programmable spectral resolution of 0.5 to $15 \mathrm{~cm}^{-1}$. The improved spectral resolution of CIRS over previous IR instrument-missions to Jupiter, the extended spectral range, and higher signal-to-noise performance provide significant advantages over previous data sets.
\end{abstract}

CIRS global observations of the mid-infrared spectrum of Jupiter at medium resolution $\left(2.5 \mathrm{~cm}^{-1}\right)$ have been analysed both with a radiance differencing scheme and an optimal estimation retrieval model to retrieve the spatial variation of phosphine and ammonia fractional scale height in the troposphere between $60^{\circ} \mathrm{S}$ and $60^{\circ} \mathrm{N}$ at a spatial resolution of $6^{\circ}$. The ammonia fractional scale height appears to be high over the Equatorial Zone (EZ) but low over the North Equatorial Belt (NEB) and South Equatorial Belt (SEB) indicating rapid uplift or strong vertical mixing in the EZ. The abundance of phosphine shows a similar strong latitudinal variation which generally matches that of the ammonia fractional scale height. However while the ammonia fractional scale height distribution is to a first order symmetric in latitude, the phosphine distribution shows a North/South asymmetry at mid latitudes with higher amounts detected at $40^{\circ} \mathrm{N}$ than $40^{\circ} \mathrm{S}$. In addition the data show that while the ammonia fractional scale height at this spatial resolution appears to be low over the Great Red Spot (GRS), indicating reduced vertical mixing above the $\sim 500 \mathrm{mb}$ level, the abundance of phosphine at deeper levels may be enhanced at the northern edge of the GRS indicating upwelling. 


\section{Introduction}

Between October 2000 and March 2001 the Cassini/Huygens spacecraft passed by Jupiter in its way to Saturn. At its closest approach on December $30^{\text {th }} 2000$, the spacecraft was 9,700,000 km away from Jupiter (136 RJ). During the fly-by, Cassini's remote sensing instruments were activated to observe Jupiter and in this paper we report the observations of Jupiter by the Composite Infrared Spectrometer (CIRS) of spectral emission features of phosphine, and adjacent or overlapping ammonia features.

Phosphine is a disequilibrium species in the cold upper tropospheres of the giant planets. Its abundance is primarily governed by temperature-dependent equilibriation reactions such as (e.g. Atreya 1986)

$$
4 \mathrm{PH}_{3}+6 \mathrm{H}_{2} \mathrm{O} \square \mathrm{P}_{4} \mathrm{O}_{6}+12 \mathrm{H}_{2} \text {, }
$$

and at the cold temperatures found in the Jovian upper troposphere its equilibrium abundance is expected to be undetectable. However the equilibration reaction (Eq. 1) is very slow, and in Jupiter's atmosphere the vertical transport of air due to convection appears to be fast enough to lead to a significant abundance in the observable troposphere of approximately $6 \times 10^{-7}$ (Kunde et al., 1982). Once in the upper troposphere, in addition to equilibration reactions, phosphine is also destroyed by solar UV photolysis to produce a number of possible species such as diphosphine $\left(\mathrm{P}_{2} \mathrm{H}_{2}\right)$. Because of these destruction processes, regions of enhanced phosphine abundance indicate rapid vertical mean uplift, or equivalently, vigorous vertical eddy mixing, which transports phosphine from the deep interior faster than it can be destroyed. The spectral absorption features of phosphine are clearly seen in Jupiter's infrared spectrum in the far-IR, mid-IR and at 5- $\mu \mathrm{m}$ wavelengths. These spectra have been used to determine that the mean abundance profile of phosphine has a 'deep' 
(i.e. at pressures greater than 1 bar) volume mixing ratio (v.m.r.) of $6 \times 10^{-7}$ (Kunde et al., 1982), and that at pressures less than 1 bar, the combined effects of vertical eddymixing, photodissociation and equilibration reactions, cause the abundance of phosphine to decrease with height with a fractional scale height (f.s.h.) of approximately 0.3 (Carlson et al., 1993; Irwin et al., 1998).

A number of studies have also been made to investigate the spatial variation of phosphine. Griffith et al. (1992) reanalysed Voyager IRIS data to determine the tropospheric abundances of phosphine and ammonia around the Great Red Spot (GRS). Since the GRS is thought to be a region of uplift at pressures greater than 500 mbar (Flasar et al., 1981; Conrath et al., 1981), it was expected that the abundances of both species should be enhanced in the GRS. However no enhancement of phosphine was observed and indeed the abundance of ammonia appeared, unexpectedly, to be depleted. Lara et al. (1998) used the NASA Infrared Telescope Facility (IRTF) to examine the thermal emission spectrum of Jupiter from $10^{\circ} \mathrm{S}$ to $40^{\circ} \mathrm{S}$ and several longitudes, between 10.5 and $11.2 \mu \mathrm{m}$. $\left(891-956 \mathrm{~cm}^{-1}\right)$. The abundances of both phosphine and ammonia were found to increase towards the equator, consistent with the Equatorial Zone (EZ) being a region of upwelling, or more rapid vertical mixing. However the abundance of ammonia was not found to vary over the GRS and while the abundance of phosphine appeared slightly enhanced, the enhancement was less than the estimated error of their retrievals. Edgington et al. (1998) analysed UV spectra observed by the HST Faint Object Spectrograph from $6^{\circ} \mathrm{N}$ to $48^{\circ} \mathrm{N}$, and deduced the same decrease in upper tropospheric ammonia abundance away from the equator observed by Lara et al. (1998). Although Edgington et al. (1998) determined the mean mixing ratio of phosphine at the $140-\mathrm{mb}$ level they were unable to extract any latitudinal dependence. 
Meridional variation in the abundance of ammonia at deeper levels has been inferred from ground-based radio observations by de Pater (1986) at a wavelength of approximately $2 \mathrm{~cm}$. A clear latitudinal variation of brightness temperature was observed and interpreted as being due to a variation in the ammonia v.m.r. between 0.5 and 2 bars of $\sim 2 \times 10^{-5}$ in the Equatorial Belts, increasing to $5 \times 10^{-5}$ in the EZ.

In this paper the spectra recorded by the Composite Infrared Spectrometer (CIRS) during Cassini's fly-by of Jupiter are analysed to retrieve, for the first time, the spatial distribution of both phosphine and ammonia in the upper troposphere at almost all longitudes and from $60^{\circ} \mathrm{S}$ to $60^{\circ} \mathrm{N}$ latitude at a spatial resolution of $6^{\circ}$. The retrieved maps show a clear latitudinal structure of both phosphine and ammonia, indicating variations in vertical transport, and also indicate a depletion of upper level ammonia, and a possible slight enhancement of deep phosphine, over the GRS.

\section{CIRS observations.}

CIRS (Calcutt et al., 1992; Kunde et al., 1996), a successor of the versatile Infrared Spectrometer and Radiometer (IRIS) instruments flown on Voyagers 1 and 2, is a coordinated pair of interferometers designed to measure infrared radiation from 10 to $1400 \mathrm{~cm}^{-1}(1000$ to $7 \mu \mathrm{m})$ with a variable spectral resolution that can be set from 0.5 to $15 \mathrm{~cm}^{-1}$. The two interferometers are situated opposite each other across the central axis of the instrument and jointly share the received light from the $50-\mathrm{cm}$ diameter telescope situated outside and in front of the instrument housing.

The mid-infrared interferometer is a classic Michelson design and has two different focal planes (FP3: $600-1100 \mathrm{~cm}^{-1}$, FP4: $1100-1400 \mathrm{~cm}^{-1}$ ) each consisting of $1 \times 10$ arrays of square photoconductive, and photovoltaic detectors respectively with individual fields-of-view (FOV) of $0.27 \mathrm{mrad}$. The small field-of-view and high 
spectral resolution of the mid-infrared channels require that the detectors be cooled to $80 \mathrm{~K}$ via an external radiator which greatly increases their sensitivity compared to the IRIS detectors. The mirror system of the mid-infrared interferometer utilizes cornercube reflectors to make the interferometer more rugged and less prone to misalignment.

The second interferometer operates in the far-infrared $\left(10-600 \mathrm{~cm}^{-1}\right)$ and is a polarizing interferometer with a circular FOV of $4.3 \mathrm{mrad}$ (just like Voyager IRIS). The far-infrared interferometer is a Martin-Puplett type which' uses polarizing wire grids and a pair of bolometers to separate and recombine the different polarizations of the incoming signal thereby removing the DC component from the resulting interferogram. This makes the spectrometer less susceptible to instrumental drifts and increases the measurement precision.

Because both interferometers share the same mirror and drive assembly, the maximum spectral resolution $\left(0.5 \mathrm{~cm}^{-1}-\right.$ almost a factor of ten better than that achieved by Voyager IRIS) is the same for both interferometers and is dependent upon the scan length.

Cassini-Huygens, a joint NASA-ESA mission, was launched in 1997 on a circuitous path utilising gravity-assists from Venus, Earth and Jupiter to propel itself towards its final destination of the Saturnian system, where it will arrive in July 2004. For the six months between 1 October 2000 and 22 March 2001 during Cassini's flyby of Jupiter the Composite Infrared Spectrometer registered more than 850,000 spectra of the Jovian atmosphere, satellites and rings. Of particular value are the ATMOS02 Jovian atmospheric observations, of which there are four in total: A, B, C and D, which were recorded during and immediately after closest approach (see Table 1). In these observations, the FP3 and FP4 detector arrays were aligned parallel to the 
equator of Jupiter and cyclically scanned from North to South Pole at medium spectral resolution $\left(2.5 \mathrm{~cm}^{-1}\right.$, apodised). Each scan lasted approximately 40 minutes and was followed by observations of other instruments. Hence successive scans did not overlap but were separated by $45^{\circ}$ longitude. Complete unbroken coverage was possible only by observing Jupiter for two complete rotations and in practice gaps occurred in all four maps due to competition for observation time with other instruments on the Cassini spacecraft.

\section{Forward Models}

The radiative transfer, or forward model used in this study is a development of code previously used to analyse Galileo/NIMS observations of Jupiter (Irwin et al., 1998, 2001). The code has been converted to run in wavenumber rather than wavelength space and uses the method of correlated- $k$ (Lacis and Oinas, 1991). Although the forward model may include scattering (for which correlated- $k$ is especially useful) the effects of scattering were found to be negligible for model CIRS spectra and have been neglected here. The code has been validated against a number of line-by-line models and is found to produce similar results but is very much faster. The small differences between synthetic correlated- $k$ and line-by-line spectra were included in the error budget of the retrieval model presented later.

The forward model uses pre-tabulated $k$-distribution data which are calculated from line-by-line models for each individual gas contributing to the CIRS spectra. The tables cover fifteen temperatures equally spaced between 100 and $350 \mathrm{~K}$, and fifteen pressures, equally spaced in $\log$ pressure between $1.7 \times 10^{-5}$ and 7.5 bar. The gas line data is based upon GEISA (Jacquinet-Husson et al., 1999) with a number of modifications for line widths and line strengths. In particular the lines for phosphine 
were corrected in the $v_{4}$ band $\left(1121 \mathrm{~cm}^{-1}\right)$ following Lellouch et al. (2001). The $k$ tables were calculated from 0 to $1500 \mathrm{~cm}^{-1}$ in square bins of width $0.25 \mathrm{~cm}^{-1}$, and were smoothed to lower resolutions as required. The collision-induced absorption of $\mathrm{H}_{2}-\mathrm{H}_{2}$ and $\mathrm{H}_{2}-\mathrm{He}$ was also included after Borysow et al. $(1985,1988)$.

The Jovian atmospheric temperature profile is represented at 71 pressure levels equally spaced in $\log$ pressure between 4 and $4 \times 10^{-7}$ bar. The volume mixing ratio (v.m.r.) of each gas is either fixed at all altitudes for constituents such as methane and helium, or allowed to vary as follows. Ammonia is assumed to have a fixed v.m.r. of $x_{0}$ at pressures greater than $p_{0}=0.7 \mathrm{bar}$ (the approximate condensation level) and to vary at lower pressures as

where $f$ is the fractional scale height (f.s.h.). In addition the ammonia profile was constrained to have a maximum relative humidity of $100 \%$. The profile of phosphine was similar but here the deep pressure $p_{0}$ was set at $1 \mathrm{bar}$, consistent with previous studies mentioned earlier, and there was no constraint on humidity. Cloud opacity was also included and modelled as a single cloud based at 1 bar with variable opacity and a fractional scale height of 0.5 in rough agreement with the cloud found by the probe nephelometer (Ragent et al., 1998) and by Galileo/NIMS and Galileo/SSI cloud retrievals (Irwin et al., 2001; Banfield et al., 1998, Simon-Miller et al., 2001b). The cloud opacity was assumed to be grey (i.e. constant absorption with wavelength) over the limited wavelength ranges considered in the retrievals.

Figure 1 shows a synthetic reference CIRS spectrum in $750-1230 \mathrm{~cm}^{-1}$ region covering most of the range of FP3 and part of FP4 using the a priori temperature, abundance and cloud assumptions listed in Table 2. Also plotted in Figure 1 are the partial derivatives of the spectral radiance with respect to ammonia and phosphine 
(deep v.m.r. and f.s.h.) and also cloud opacity. The altitudes sounded in the mid-IR range from 0.2 to approximately 1 bar as may be seen in Figure 2 which shows how the peaks of the transmission weighting functions and contribution functions (i.e. the transmission weighting functions multiplied by thermal emission of the atmosphere at each level and wavelength) vary with wavenumber. Comparing Figures 1 and 2 it can be seen that the wavelengths where the spectrum shows significant sensitivity to phosphine (both deep and f.s.h.) correspond to weighting functions peaking near the 1 bar level and thus this is the pressure level of the phosphine profile that the mid-IR spectrum is most sensitive to. More quantitatively, over the $1000-1200 \mathrm{~cm}^{-1}$ wavenumber range used in the retrievals presented later, the average transmission weighting function, weighted by the sensitivity to deep phosphine abundance, peaks at $0.88 \mathrm{bar}$, and the mean contribution function peaks at 1.2 bar. Similarly, the average transmission weighting functions weighted by the sensitivity to phosphine f.s.h., deep ammonia abundance, and ammonia f.s.h. peak at $0.82,0.75$, and 0.64 bar respectively. Corresponding values for the mean contribution function peaks are $1.1,0.94$, and 0.76 bar. Hence information on the ammonia abundance comes from higher altitudes than for the phosphine profile. It should be noted that the reference spectrum was calculated for a cloud-opacity of 0.5 above 1 bar. In more cloudy regions, the weighting functions move up and thus the abundance information comes from correspondingly higher altitudes.

Most of the radiance partial derivative curves in Figure 1 (and indeed the radiance partial derivatives due to the temperature at different levels, which are not shown) are very different from each other and thus many different parameters may be independently determined. The main exception is for the two phosphine parameters for which the dependence on deep abundance and fractional scale height are found to 
be very similar and in effect indistinguishable. This is due to the absorption lines of phosphine only appearing in a limited spectral region, sounding near the 1 bar level. Hence the f.s.h. was fixed at the a priori value of 0.3 , consistent with a number of previous studies (Carlson et al., 1993; Irwin et al., 1998) and only the deep v.m.r. was fitted in the retrievals presented later.

\section{Radiance Differencing}

Retrieving atmospheric composition from remotely sensed spectra is a classic illposed problem in that many possible atmospheric profiles generate spectra which are consistent with the observed spectra, due to uncertainties both in the spectra and in the forward model. Hence before analysing the data with complicated retrieval models, it was decided to first of all see whether the raw spectra could be simply analysed to provide a qualitative indication of the spatial distribution of phosphine.

The nominal synthetic spectrum and radiance partial derivative curves of Figure 1 were searched for pairs of wavelengths of equal brightness temperature (and thus sounding roughly the same pressure level) which were equally affected by changes in temperature, ammonia (both deep volume mixing ratio and fractional scale height) and cloud opacity, but differentially affected by variations in the phosphine abundance. Pairs with small wavelength differences were preferred over those with large wavelength differences since the assumption of grey cloud absorption was then more valid. Pairs of wavelengths with a brightness temperature of $154 \mathrm{~K}$ (sounding roughly the 0.7 bar level) in the synthetic spectrum were analysed in this way, and the optimum pair was found to be $\left(1186.7 \mathrm{~cm}^{-1}, 1190 \mathrm{~cm}^{-1}\right)$. Figure 3 shows the reference synthetic spectrum in the region of this pair together with the differences introduced by varying the a priori ammonia (deep and f.s.h), phosphine (deep and f.s.h.) and 
cloud opacities by $50 \%$. It can clearly be seen that the brightness temperatures at the two wavelengths (indicated by ' + ' symbols) vary by a similar amount for all parameters (including temperatures which are not shown here) except phosphine for which very different responses are seen. Hence by preparing brightness temperature maps at these two wavelengths and subtracting them, any variations observed should be attributable to phosphine variation alone.

To demonstrate the validity of this technique, a set of test spectra were first calculated. Since we wanted a representative range of conditions for all latitudes between $60^{\circ} \mathrm{S}$ and $60^{\circ} \mathrm{N}$, temperature profiles were first retrieved (with a separate model) from the ATMOS02A data set from $55^{\circ} \mathrm{S}$ to $55^{\circ} \mathrm{N}$ averaged into latitudinal bins of width $10^{\circ}$ and step $5^{\circ}$, giving 23 different temperature profiles. These profiles were retrieved from apodised spectra (Hamming instrument function, FWHM $=2.5$ $\mathrm{cm}^{-1}$ ) in the range $600-700 \mathrm{~cm}^{-1}$. For each of the 23 assumed temperature profiles and observing geometry conditions, the following atmospheric state parameters were then randomly varied and a number of sample synthetic spectra calculated:

$\mathrm{NH}_{3}$ deep fraction (relative to an assumed solar value of $2.19 \times 10^{-4}$ ) set to $1.0+$ $0.5 x$, where $x$ is a random number between \pm 1 .

$\mathrm{NH}_{3}$ fractional scale height set to $0.15+0.2 x$ (limited not to go below 0.01 ).

Cloud base set to 1 bar, with fractional scale height 0.5 , and optical depth set to $0.3+0.5 x$ (limited not to go below 0.0 ).

$\mathrm{PH}_{3}$ deep fraction (relative to $6 \times 10^{-7}$ ) set to $1.0+0.5 x$. Fractional scale height fixed at 0.3 .

(n.b. the random number $x$ was recalculated for each element) 
For each temperature profile, four random sets of the above parameters were generated and used to calculate $23 \times 4=92$ synthetic spectra in total. The spectral resolution of the forward model was set to be triangular with a full-width-half maximum of $2.5 \mathrm{~cm}^{-1}$, found to be a convenient and sufficiently accurate approximation to the real instrument function.

Using these test spectra the value of $\Delta T_{B}=T_{B}\left(1190 \mathrm{~cm}^{-1}\right)-T_{B}\left(1186.7 \mathrm{~cm}^{-1}\right)$ was plotted against the values of the ammonia, phosphine, cloud and temperature parameters used to generate the spectra. The sign of $\Delta T_{B}$ was chosen to ensure that positive $\Delta T_{B}$ values correspond to enhanced abundances of phosphine. The results for the ammonia, phosphine and cloud parameters are shown in Figure 4 and a clear correlation with phosphine abundance is seen, while there is no correlation with any other parameter (including the temperatures at different altitudes which are not shown here).

The same procedure was then applied to the ATMOS02A dataset and the brightness temperature maps at the two reference wavelengths, and difference, are shown in Figure 5. The zonal structure of Jupiter is clearly seen in the brightness temperature maps where the zones are cold and where the GRS is clearly seen at $50^{\circ}$ $\mathrm{W}, 25^{\circ} \mathrm{S}$. The dark near-vertical stripes are due to missing data. In practice it was found that there was a small variation in the wavelength calibration of this, and the three other ATMOS02A maps, and thus the wavelengths were slightly adjusted to coincide with the either side of the $1187.5 \mathrm{~cm}^{-1}$ brightness temperature peak. Figure 6 shows the adjusted $\Delta \mathrm{T}_{\mathrm{B}}$ signal for all four maps. The missing data swaths are clearly obvious in all four maps, and it can also be seen that calibration problems remain with the ATMOS02B set caused, it is believed, by changing instrument temperature which clearly affect the radiometric calibration. However in all four cases a clear meridional 
variation of the signal is seen, together with a slight possible enhancement over the

GRS. Figure 7 shows an average $\Delta T_{B}$ map for the $A, C$ and $D$ ATMOS02 observations (the $\mathrm{B}$ observation was neglected due to remaining calibration problems). These averaged data show more clearly the increased $\Delta T_{B}$ values (and hence higher $\mathrm{PH}_{3}$ abundance) over the EZ, decreased values over the North Equatorial Belt (NEB) and South Equatorial Belt (SEB), and a possible local maximum over the GRS. In addition, there is also an indication of North/South asymmetry in phosphine abundance at mid-latitudes, with increased abundances seen in the northern hemisphere.

\section{Retrieval Model}

The radiance differencing method of the previous section provides a qualitative indication of the distribution of phosphine, but only uses two wavelengths and thus is rather sensitive to noise. To provide a better-averaged map, and also to provide a more quantitative analysis, a non-linear optimal estimation retrieval algorithm after Rodgers $(1976,2000)$ was applied. The scheme minimises the difference between measured and modelled spectra, subject to minimum departure from the a priori vector, by minimising the 'cost' function:

where

y is the measured spectrum.

is the spectrum calculated with the forward model.

$\mathbf{S}_{\varepsilon} \quad$ is the measurement covariance matrix, which contains both estimated measurement errors and forward modelling errors. 
$\mathbf{x} \quad$ is the model state vector.

a is the a priori state vector.

$S_{x} \quad$ is the a priori covariance matrix.

The diagonal components of the a priori covariance matrix were set to the square of the estimated a priori errors. Off-diagonal elements were set to zero except for the temperature parameters, where a correlation length (in $\log$ (pressure) units) of 1.5 was adopted to provide vertical smoothing (e.g. Rodgers $2000, \mathrm{p} 55$ ). In addition to the constraints defined by the $a$ priori assumptions, further 'hard limits' were set to eliminate non-physical solutions. All the error values and limits used are listed in Table 2. Together with the a priori temperature error reducing with depth in the atmosphere to force barotropic conditions at higher pressures, a further constraint was placed on the temperature profile below the tropopause which was prevented from decreasing more rapidly with height than the dry adiabatic lapse rate (DALR).

Given these constraints, at each step in the iterative retrieval process a new estimate of the state vector $\mathbf{x}$ was estimated from optimal estimation theory to be

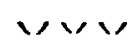

where $\mathbf{K}_{n}$ is the matrix containing the rates of change of the $n^{\text {th }}$ calculated spectrum $y_{n}$ with respect to all the elements in the state vector $\mathbf{x}$. However, to stop the model becoming unstable, the actual modified state vector used, $\mathbf{x}_{n+1}^{\prime}$, was adjusted slightly using a Marquardt-Levenberg-type parameter (Press et al., 1992) $\lambda$ as: 
The parameter $\lambda$ was initially set to 1.0 . If the spectrum calculated from $\mathbf{x}_{n+1}^{\prime}$ was found to reduce the cost function $\phi$, then $\mathbf{x}_{\mathbf{n}}$ was set to $\mathbf{x}_{n+1}^{\prime}, \lambda$ was multiplied by a factor of 0.3 , and the next iteration started. If however the spectrum calculated from $\mathbf{x}_{n+1}^{\prime}$ was found to increase the cost function $\phi$, then $\mathbf{x}_{\mathrm{n}}$ was left unchanged, the parameter $\lambda$ increased by a factor of 10 and a new vector $\mathbf{x}_{n+1}^{\prime}$ calculated. The choice of the multiplication parameters $(0.3,10)$ is somewhat arbitrary although it is important to ensure that they are not reciprocals of one another which can lead to endless loops. To ensure smooth convergence the lower number $(0.3)$ was chosen to be greater than the reciprocal of the larger number so that $\lambda$ did not decrease too quickly. As the retrieval approaches its final solution, $\lambda \rightarrow 0$ and the model tends to the optimal estimate.

To test the joint retrieval of tropospheric temperature, ammonia, phosphine and cloud (or haze) opacity, the retrieval model was applied to the set of synthetic spectra discussed earlier. Each spectrum was first cut to leave only the $600-700 \mathrm{~cm}^{-1}$, and $1000-1200 \mathrm{~cm}^{-1}$ ranges. The noise on these synthetic spectra was set to the estimated Noise Equivalent Spectral Radiance (NESR) of the spectra, reduced by factors appropriate for the number of spectra averaged into the $10^{\circ}$-wide latitude bins. Forward modelling errors were also added at this stage to account for possible systematic errors in the forward model. The retrieval model was then run on each case for a maximum of 10 iterations or until the minimisation function $\phi$ had converged to within $0.5 \%$.

The results of this retrieval run for the composition and cloud parameters are shown in Figure 8. This figure shows the correlation between the true and retrieved values of ammonia (deep fraction and f.s.h.), deep phosphine fraction and cloud (or haze) opacity for all 92 cases. The a priori values are indicated by the dashed lines 
and the $a$ priori errors by the dotted lines. The fitted values are indicated by + signs, with the vertical extent set to the estimated retrieval error of the model calculated from:

It can be seen that in most cases, the retrieved parameters fit the 'true' parameters to within the $1-\sigma$ retrieval error calculated from Eq. 6, validating the correct behaviour of the retrieval model. The peculiar appearance of the ammonia fractional scale height plot at high f.s.h. values arises from some of the random combinations of the two ammonia parameters leading to saturation of ammonia at higher levels in the synthetic profiles. Since the volume mixing ratio (v.m.r.) of ammonia is limited not to go above the saturated v.m.r. this means that in these cases the ammonia profiles used to generate the synthetic spectra have lower ammonia concentrations at pressures below $700 \mathrm{mb}$ than prescribed by the ammonia parameter combinations alone. Hence the ammonia v.m.r. profile used to generate these synthetic spectra is actually better approximated by a different combination of the two ammonia parameters, with a lower fractional scale height, which the retrieval model correctly deduces.

\section{Global retrievals.}

The spectra in the ATMOS02A map were analysed using this retrieval model between 600 and $700 \mathrm{~cm}^{-1}$ and $1000-1200 \mathrm{~cm}^{-1}$ to retrieve jointly the temperature profile, the deep phosphine abundance, the deep ammonia abundance and fractional scale height, and the total cloud (or haze) opacity. The fractional scale height of phosphine was fixed at 0.3 as described earlier. The spectra were averaged into bins of width $6^{\circ}$ in both latitude and longitude and stepped every $3^{\circ}$ in longitude between 0 and $360^{\circ} \mathrm{W}$ 
and between $60^{\circ} \mathrm{S}$ and $60^{\circ} \mathrm{N}$ to achieve Nyquist sampling. The error on these average spectra was then calculated including the averaged NESR, sample variation, and a very conservative estimate of the forward modelling error of $10 \%$. The temperature retrievals were found to be in good agreement with the temperature retrievals of previous CIRS analyses (Flasar et al., 2003). Similarly the ammonia fractional scale height (f.s.h.) retrievals are consistent with those of Fouchet et al. (2003), who considered a slightly different wavenumber range $\left(900-940 \mathrm{~cm}^{-1}\right)$. For the deep ammonia retrievals however, the variation across the planet was found to be insignificant compared to the retrieval error and thus nothing could be inferred. Similarly, the results of the cloud (or haze) opacity retrievals were found to depend strongly on the a priori assumptions. An atmosphere with a cloud of uniform absorption cross-section generates a thermal emission spectrum which is virtually indistinguishable over small wavelength ranges from a cloud-free atmosphere with a suitably adjusted temperature profile. Hence the only way that both the temperature profile and cloud opacity can be independently retrieved is by making assumptions on the vertical profile of the cloud, and placing suitable constraints on the T-profile (e.g. Conrath and Gierasch, 1986). Hence in this case, these variables may be thought of simply as free parameters, used to minimise the difference between the synthetic and measured spectra, in regions away from phosphine absorption features. The exception to this is the ammonia scale height retrieval which is well constrained by these data and whose spatial variations are large compared to the retrieval errors.

Figure 9 shows the global variation of the retrieved fractional scale height of ammonia, and the 'deep' phosphine abundance. The scale bars in Figure 9 also show the a priori and retrieved errors of the individual retrievals and it can be seen that the ammonia f.s.h. is very well constrained by the data, but the phosphine abundance less 
so. The ammonia f.s.h. shows clear latitudinal variation with higher values in the EZ and lower values in the North Equatorial Belt (NEB) and South Equatorial Belt (SEB), consistent with the canonical view of upwelling of moist, volatile-rich air of the zones and subsidence over the belts (Stone, 1976). It is also apparent that the ammonia f.s.h. appears depleted over the centre of the GRS which is consistent with previous investigations (e.g. Griffith et al. 1992) and suggests stagnation of the air at levels above $\sim 500$ mbar. The meridional pattern of the ammonia f.s.h. distribution is also repeated in the deep phosphine abundance map which shows clear enhancement over the EZ and depletion over the SEB and NEB. However, while $\mathrm{PH}_{3}$ appears to be enhanced at $\sim 40^{\circ} \mathrm{N}$, no clear enhancement is seen at $40^{\circ} \mathrm{S}$. The cause of this asymmetry is unclear but was also observed in the brightness temperature difference maps shown earlier. An intriguing difference between the phosphine and ammonia maps is that while ammonia f.s.h. is depleted in the centre of the GRS the abundance of phosphine appears to be slightly enhanced at the northern edge. To assess the significance of this, the variation of the retrieved phosphine abundance, and retrieval errors are shown for a longitude strip (Figure 10) passing through the GRS and also a latitude strip (Figure 11). While the GRS signature in the single latitude strip could, on its own, be easily discarded as noise, the GRS signature in the longitude strip looks at first sight more convincing. In this longitude strip one hundred estimates of the phosphine abundance were made. The mean value was found to be $1.04 \times 10^{-6}$ with a standard deviation of $1.02 \times 10^{-7}$. Using this error value, the two points in the GRS at $48^{\circ}$ and $51^{\circ} \mathrm{W}$ are found to be over four standard deviations away from the mean. Assuming Gaussian statistics, the probability of finding such a point is only $6.3 \times 10^{-5}$ and thus in a hundred samples the number of such points is expected to be much less than one $(0.0063)$. Hence on this basis the identification of increased phosphine 
abundance over the GRS would appear to be statistically significant. However this analysis neglects the retrieval errors for which the mean value was found to be $2.3 \times$ $10^{-7}$. Substituting this as the standard deviation, the two GRS points are then found to be just below two standard deviations from the mean for which the probability is 0.045 . Hence in one hundred samples, four such points might be expected and thus the identification of phosphine enhancement over the GRS looks rather less secure. It should be remembered that a very conservative forward modelling error of $10 \%$ has been assumed in these retrievals to ensure smooth maps. When a smaller value of $5 \%$ is assumed, the retrieved phosphine map looks much noisier and the GRS signature is much harder to discern although the same mean latitudinal variation is seen. An additional factor to consider is the calibration of the CIRS dataset for which work is still on-going. The apparent $\mathrm{PH}_{3}$ signature over the GRS is found to be more visible in some versions of the calibrated data than others, and thus the results presented here should be considered as first indications rather than definitive final values. With the current state of the data set and modelling, the most the can be said at present is that the radiative differencing and retrieval models both show a clear latitudinal variation of phosphine, with North/South asymmetry, and an indication that phosphine may be enhanced over the northern edge of the GRS.

\section{Conclusions.}

The spatial distribution of phosphine, and also ammonia fractional scale height (f.s.h.) in Jupiter's atmosphere has been retrieved from $60^{\circ} \mathrm{S}$ to $60^{\circ} \mathrm{N}$ with high spatial resolution and low noise using Cassini CIRS spectra. We find that both ammonia f.s.h. and phosphine abundance are enhanced over the Equatorial Zone and depleted over the North and South Equatorial Belts in agreement with the canonical view of 
upwelling in the zones and subsidence in the belts (Stone, 1976). It should be noted however that the same effect may be achieved if vertical eddy mixing is more active over the zones and the observation does not necessarily imply bulk overturning motion. The behaviour at more polar latitudes is more puzzling in that while the latitudinal variation in ammonia f.s.h. appears symmetric, a noticeable North/South asymmetry in phosphine abundance is observed at mid-latitudes $\left(\sim 40^{\circ}\right)$. The origin of this asymmetry is unknown although is presumably linked to the other well-known asymmetries in the Jovian atmosphere including the prevalence of large-scale anticyclonic eddies such as the GRS in the southern hemisphere.

It has also been possible to determine the possible variation in abundance of both these volatiles over the GRS. Like Griffith et al. (1992) we find that upper level ammonia is depleted over the GRS, presumably due to a mixture of condensation and photodissociation. Phosphine however, sounded at slightly deeper levels, appears to be enhanced as was noted by Lara et al. (1998), although this conclusion is right on the edge of our detection limits. The apparent difference in behaviour between ammonia and phosphine over the GRS is at first glance rather confusing. However it should be remembered that the ammonia abundance is sampled at higher altitudes than phosphine at these wavelengths. Hence the high abundance of deep phosphine and the small ammonia fractional scale height are consistent with the canonical model of the GRS being an area of uplift at deeper levels but somewhat stagnant at upper levels (Flasar et al., 1981; Conrath et al., 1981) allowing of time for condensation, rain-out and photodissociation, thus reducing the abundance of higher altitude ammonia. This interpretation is also consistent with observation that the deep phosphine is possibly enhanced at the northern edge of the GRS, while the low ammonia f.s.h. values are found further south. From temperature retrievals the centre 
of the GRS at high altitudes is found to be considerably further south than at lower altitudes. Hence the high phosphine values (retrieved at deeper levels) are found to coincide with the centre of the GRS at deep levels while the low ammonia f.s.h. values (sounded at much higher altitudes) coincide with the centre at higher altitudes. It should be noted here that these results are dependent on spatial resolution. In order that we can detect phosphine we have used quite wide latitude/longitude bins in these retrievals to reduce noise. However such large bins may smear out localised features. Models retrieving ammonia only may use smaller latitude/longitude bins to achieve sufficient signal-to-noise ratios and such models developed at NASA/GSFC (Achterberg, private communication) to analyse the same ATMOS02 data find that while ammonia is depleted in the southern part of the GRS, as seen here, it appears enhanced in small areas to the north.

Prior to the Cassini Jupiter encounter, it was hoped that CIRS spectra might help to identify the chromophores in Jupiter's clouds. In particular, it had been suggested (e.g. Prinn and Lewis 1975) that triclinic red Phosphorus $\left(\mathrm{P}_{4}\right)$, a possible product of phosphine photolysis, might be responsible for the colour of the GRS. However a number of other authors argue against this identification. West et al. (1986) found that both the red and white allotropes of Phosphorus are too blue-absorbing to match the observed redness of the GRS and instead Sulphur $\left(\mathrm{S}_{8}\right)$ allotropes seemed to do better. In addition Boudon et al. (1999) found that white phosphorus has a strong absorption feature at $466 \mathrm{~cm}^{-1}$, which should also be present in red phosphorus and would be visible in the far-infrared CIRS spectra. However no such features have been detected. Finally Simon-Miller et al. (2001a) have performed a Principal Component Analysis of HST Jupiter observations and conclude that the anomalous redness of the GRS, compared to other red regions, is likely only a trace constituent whose actual identity 
remains a mystery. Laboratory spectra of sulphur and phosphorus allotropes, as well as higher-order hydrocarbons and other photochemical products like $\mathrm{P}_{2} \mathrm{H}_{2}$ are needed from the near-UV through the far-infrared to aid in this identification.

\section{Acknowledgements.}

The UK authors acknowledge the support of the Particle Physics and Astronomy Research Council. We are also deeply indebted to our colleagues at NASA Goddard Space Flight Center and to our reviewers for their very helpful moments.

\section{References}

Atreya, S.K. 1986. Atmospheres and Ionospheres of the Outer Planets and Their Satellites. Springer-Verlag, Heidelberg, Germany.

Baines, K.H., R.W. Carlson, G.S. Orton, and the Galileo NIMS Team 1996. The vertical and dynamical structure of the Great Red Spot as determined by Galileo/NIMS. Bull. Am. Astron. Soc. 28., 1136. 28th DPS Meeting Abstracts, Tucson, AZ.

Banfield, D., P.J. Gierasch, M. Bell, E. Ustinov, A.P. Ingersoll, A.R. Vasavada, R. West and M.J.S. Belton 1998. Jupiter's cloud structure from Galileo imaging data. Icarus 135, 230-250.

Borysow, J., L. Trafton, L. Frommhold and G. Birnbaum 1985. Modelling of pressure-induced far-infrared absorption spectra: Molecular hydrogen pairs. Astrophys. J. 296, 644-654.

Borysow, J., L. Frommhold and G. Birnbaum 1988. Collision-induced rototranslational absorption spectra of $\mathrm{H}_{2}$ - $\mathrm{He}$ pairs at temperatures from 40 to $3000 \mathrm{~K}$. Astrophys. J. 326, 509-515. 
Boudon, V., E.B. Mkadmi, H. Bürger and G. Pierre 1999. High-resolution Fourier transform infrared spectroscopy and analysis of the $v_{3}$ fundamental band of $\mathrm{P}_{4}$. Chem. Phys. Lett. 305, 21-27.

Calcutt, S., F. Taylor, P. Ade, V. Kunde, and D. Jennings 1992. The Composite Infrared Spectrometer. J. British Interplan. Soc. 45, 811-816.

Carlson, B., A. Lacis and W. Rossow 1993. Tropospheric Gas Composition and Cloud Structure of the Jovian North Equatorial Belt. J. Geophys. Res. 98, 52515290.

Conrath, B.J., F.M. Flasar, J.A. Pirraglia, P.J. Gierasch and G.E. Hunt 1981. Thermal Structure and Dyanmics of the Jovian Atmosphere 2. Visible Cloud Features. $J$. Geophys. Res. 86, 8769-8775.

Conrath, B.J. amd P.J. Gierasch 1986. Retrieval of ammonia abundances and cloud opacities on Jupiter from Voyager IRIS spectra. Icarus 67, 444-455.

de Pater, I. 1986. Jupiter's zone-belt structure at radio wavelengths. Icarus 68, 344365.

Dyudina, U.A., A.P. Ingersoll, G.E. Danielson, K.H. Baines, R.W. Carlson, and the Galileo NIMS and SSI Teams 2001. Interpretation of NIMS and SSI images on Jovian cloud structure. Icarus 150, 219-233.

Edgington, S.G., S.K. Atreya, L.M. Trafton, J.J. Caldwell, R.F. Beebe, A.A. Simon, R.A. West, and C Barnet 1998. On the latitude variation of ammonia, acetylene, and phosophine altitude profiles on Jupiter from HST Faint Object Spectrograph observations. Icarus 133, 192-209.

Flasar, F.M., B.J. Conrath, J. Pirraglia, P.C. Clark, R.C. French, and P.J. Gierasch 1981. Thermal structure and dynamics of the Jovian atmosphere. I - The Great Red Spot, J. Geophys. Res. 86, 8759-8767. 
Flasar, F.M. et al. 2003. Prospecting Jupiter in the infrared: temperatures and dynamics. Nature (in press).

Fouchet, T., P.G.J. Irwin, P. Parrish, S.B. Calcutt, F.W. Taylor, F. M. Flasar 2003. Search for spatial variations in the Jovian ${ }^{15} \mathrm{~N} /{ }^{14} \mathrm{~N}$ ratio from Cassini/CIRS observations. Icarus (this issue).

Griffith, C.A., B. Bézard, T. Owen and D. Gautier 1992. The tropospheric abundances of $\mathrm{NH}_{3}$ and $\mathrm{PH}_{3}$ in Jupiter's Great Red Spot from Voyager IRIS Observations. Icarus 98, 82-93.

Irwin, P.G.J., A.L. Weir, S.E. Smith, F.W. Taylor, A.L. Lambert, S.B. Calcutt, P.J. Cameron-Smith, R.W. Carlson, K. Baines, G.S. Orton, P. Drossart, T. Encrenaz, and M. Roos-Serote 1998. Cloud structure and atmospheric composition of Jupiter retrieved from Galileo NIMS real-time spectra. J. Geophys. Res. 103, $23,001-23,021$.

Irwin, P.G.J., A.L. Weir, F.W. Taylor, S.B. Calcutt, and R.W. Carlson 2001. The origin of belt/zone contrasts in the atmosphere of Jupiter and their correlation with 5-micron opacity. Icarus 149, 397-415.

Jacquinet-Husson, N. et al. 1999. The 1997 spectroscopic GEISA databank. J. Quant. Spectrosc. Rad. Trans. 62, 205-254.

Kunde, V., R. Hanel, W. Maguire, D. Gautier, J.P. Baluteau, A. Marten, A. Chedin, N, Husson and N. Scott 1982. The tropospheric gas composition of Jupiter's North Equatorial Belt $\left(\mathrm{NH}_{3}, \mathrm{PH}_{3}, \mathrm{CH}_{3} \mathrm{D}, \mathrm{GeH}_{4}, \mathrm{H}_{2} \mathrm{O}\right)$ and the Jovian D/H ratio. Astrophys. J., 263, 443-467.

Kunde, V. et al. 1996. Cassini infrared fourier spectroscopic investigation. Proc. Soc. Photo-Opt. Instrum. Eng. 2803, 162-177. 
Lacis, A.A. and V Oinas 1991. A description of the correlated- $k$ distribution method for modelling nongray gaseous absorption, thermal emission, and multiple scattering in vertically inhomogeneous atmospheres. J. Geophys. Res. 96, 90279063.

Lara, L.-M., B. Bézard, C.A. Griffith, J.H. Lacy and T.Owen 1998. High resolution 10-micrometer spectroscopy of ammonia and phosphine lines on Jupiter. Icarus $131,317-333$.

Lellouch, E., B. Bézard, T. Fouchet, H. Feuchtgruber, T. Encrenaz and T. de Graaw 2001. The deuterium abundance in Jupiter and Saturn from ISO-SWS observations. Astron. \& Astrophys. 670, 610-622.

Press, W.H., S.A. Teukolsky, W.T. Vetterling, and B.P. Flannery 1992. Numerical Recipes in Fortran, $2^{\text {nd }}$ Edition, Cambridge University Press.

Prinn, R. and J.S. Lewis 1975. Phosphine on Jupiter and implications for the Great Red Spot. Science 190, 274-276.

Ragent, B., D.S. Colburn, K.A. Rages, T.C.D. Knight, P. Avrin, G.S. Orton, P.A. Yanamandra-Fisher and G.W. Grams 1998. The clouds of Jupiter: Results of the Galileo Jupiter Mission Probe Nephelometer Experiment. J. Geophys. Res. 103, 22891-22909.

Rodgers, C.D. 1976. Retrieval of atmospheric temperature and composition from remote sensing measurements of thermal radiation. Rev. Geophys. Space Phys.,14, 609-624.

Rodgers, C.D. 2000. Inverse Methods for Atmospheric Sounding: Theory and Practice, World Scientific.

Simon-Miller, A.A., D. Banfield and P.J. Gierasch 2001a. An HST study of Jovian chromophores. Icarus 149, 94-106. 
Simon-Miller, A.A., D. Banfield and P.J. Gierasch 2001b. Color and the vertical structure in Jupiter's belts, zones and weather systems. Icarus 154, 459-474.

Stone, P.H. 1976. The meteorology of the Jovian atmosphere, Jupiter, Ed.: T. Gehrels, University of Arizona Press.

West, R.A., D.F. Strobel and M.G. Tomasko 1986. Clouds, aerosols and photochemistry in the Jovian atmosphere. Icarus 65, 161-217. 
Table 1. CIRS ATMOS02 global observations of the Jovian atmosphere

\begin{tabular}{|c|c|c|c|c|c|}
\hline Map Name & $\begin{array}{c}\text { Start } \\
\text { Time [UT] }\end{array}$ & $\begin{array}{c}\text { End Time } \\
\text { [UT] }\end{array}$ & $\begin{array}{c}\text { Spatial } \\
\text { Resolution } \\
\text { [degrees] }\end{array}$ & $\begin{array}{c}\text { Number } \\
\text { of FP3 } \\
\text { Spectra }\end{array}$ & $\begin{array}{c}\text { Number } \\
\text { of FP4 } \\
\text { Spectra }\end{array}$ \\
\hline ATMOS02A & 17:00 31Dec00 & 13:00 01 Jan01 & 2.17 & 7293 & 7385 \\
\hline ATMOS02B & $15: 00$ 04Jan01 & 10:00 05Jan01 & 2.44 & 7035 & 7200 \\
\hline ATMOS02C & $18: 00$ 09Jan01 & 09:00 10Jan01 & 3.08 & 4754 & 4721 \\
\hline ATMOS02D & 23:00 10Jan01 & 14:00 11Jan01 & 3.26 & 4475 & 4440 \\
\hline
\end{tabular}

Table 2. A priori values and errors used in retrieval model.

\begin{tabular}{|l|l|l|l|}
\hline Parameter & A priori value & A priori error & Hard limits \\
\hline $\begin{array}{l}\text { Deep } \mathrm{NH}_{3} \text { factor } \\
\left.\text { (ref. } 2.19 \times 10^{-4}\right)\end{array}$ & 1 & \pm 0.5 & $\begin{array}{l} \pm 2.0 \\
\text { (super-saturation } \\
\text { not allowed) }\end{array}$ \\
\hline $\begin{array}{l}\mathrm{NH}_{3} \text { fractional } \\
\text { sale height (f.s.h.) }\end{array}$ & 0.15 & \pm 0.1 & $\begin{array}{l} \pm 0.2 \\
\text { (super-saturation } \\
\text { not allowed) }\end{array}$ \\
\hline $\begin{array}{l}\text { Deep } \mathrm{PH}_{3} \text { factor } \\
\text { (ref. } 6 \times 10^{-7} \text { ) }\end{array}$ & 1 & \pm 0.5 & \pm 5.0 \\
\hline Cloud opacity & 0.5 & \pm 0.3 & \pm 2.0 \\
\hline Temperatures & $\begin{array}{l}\text { Standard T-profile. } \\
\text { Correlation length of } \\
1.5 \text { in log(pressure) }\end{array}$ & $\begin{array}{l} \pm 5 \mathrm{~K} \text { at } 4 \text { bars } \\
\text { increasing to } \pm 20 \mathrm{~K} \\
\text { at } 4 \times 10^{-7} \text { bar }\end{array}$ & $\begin{array}{l} \pm 20 \mathrm{~K} \text { at } 4 \text { bars } \\
\text { increasing to } \pm 80 \mathrm{~K} \\
\text { at } 4 \times 10^{-7} \text { bar }\end{array}$ \\
\hline
\end{tabular}


Figures

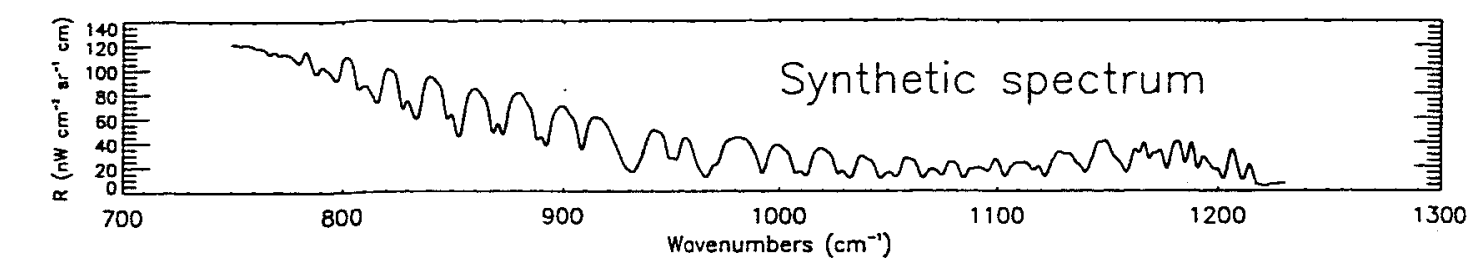

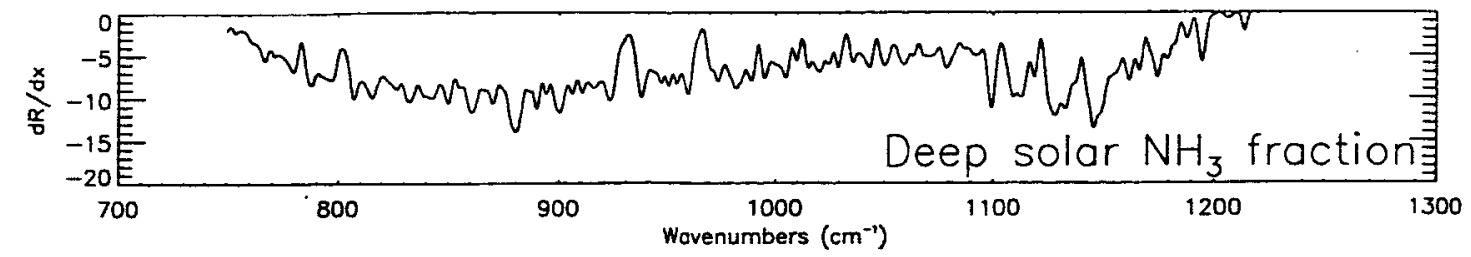
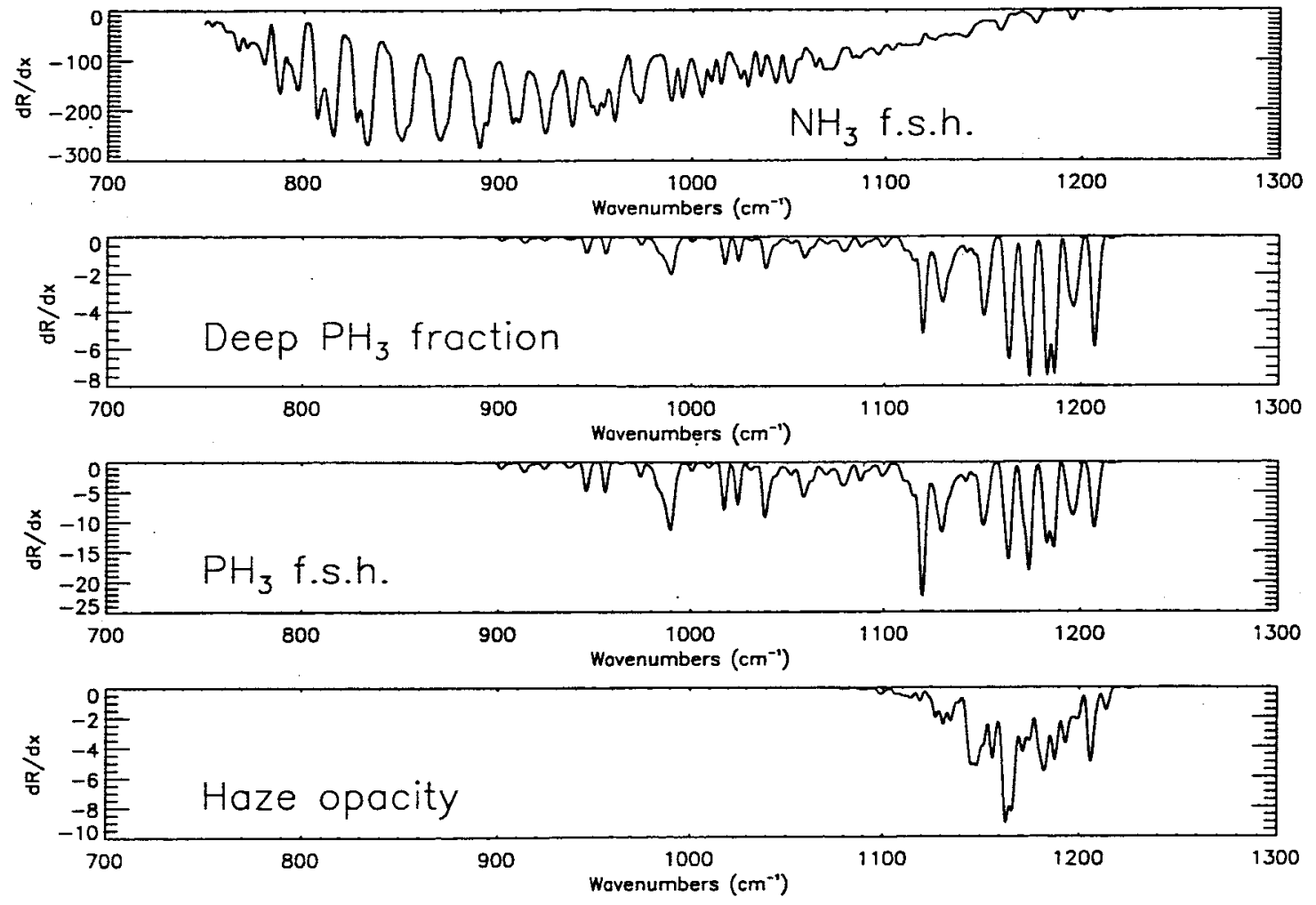

Figure 1. Synthetic nadir spectrum of Jupiter at a resolution of $2.5 \mathrm{~cm}^{-1}$ in the mid-IR range together with the rates of change of radiance with ammonia deep fractional solar abundance (reference solar volume mixing ratio taken to be $2.19 \times 10^{-4}$ ), ammonia fractional scale height (f.s.h.), phosphine deep fraction (reference v.m.r. taken to be $6.0 \times 10^{-7}$ ), phosphine f.s.h., and cloud (or haze) opacity. 


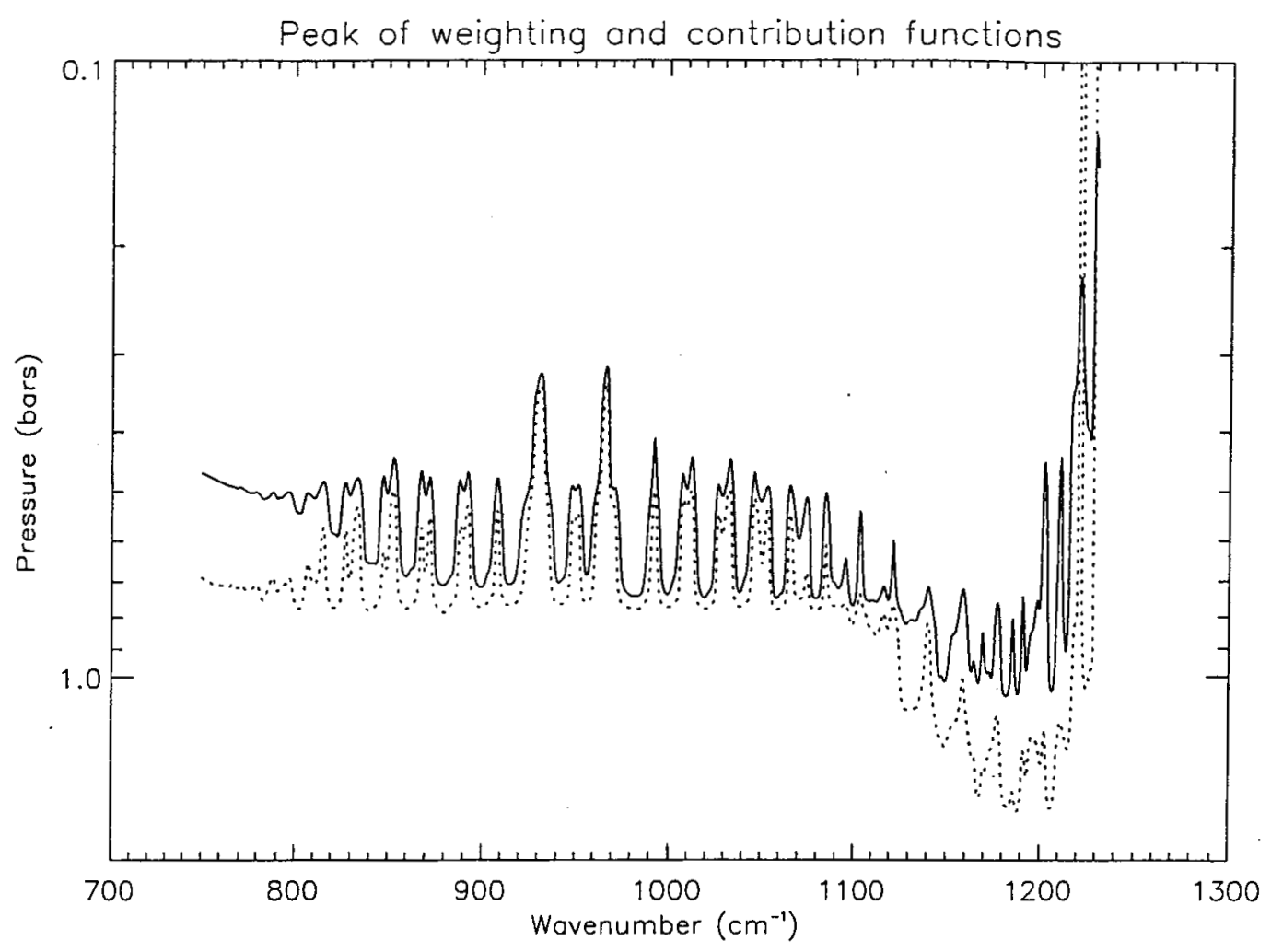

Figure 2. Variation of peak of nadir transmission weighting function (solid line) and contribution function (dotted line) as a function of wavenumber at a resolution of 2.5 $\mathrm{cm}^{-1}$ for the a priori Jovian atmosphere which includes a cloud (or haze) layer based at 1 bar with an opacity of 0.5 . N.B. the contribution function is the transmission weighting function multiplied by the atmospheric thermal emission at each level and wavenumber. 

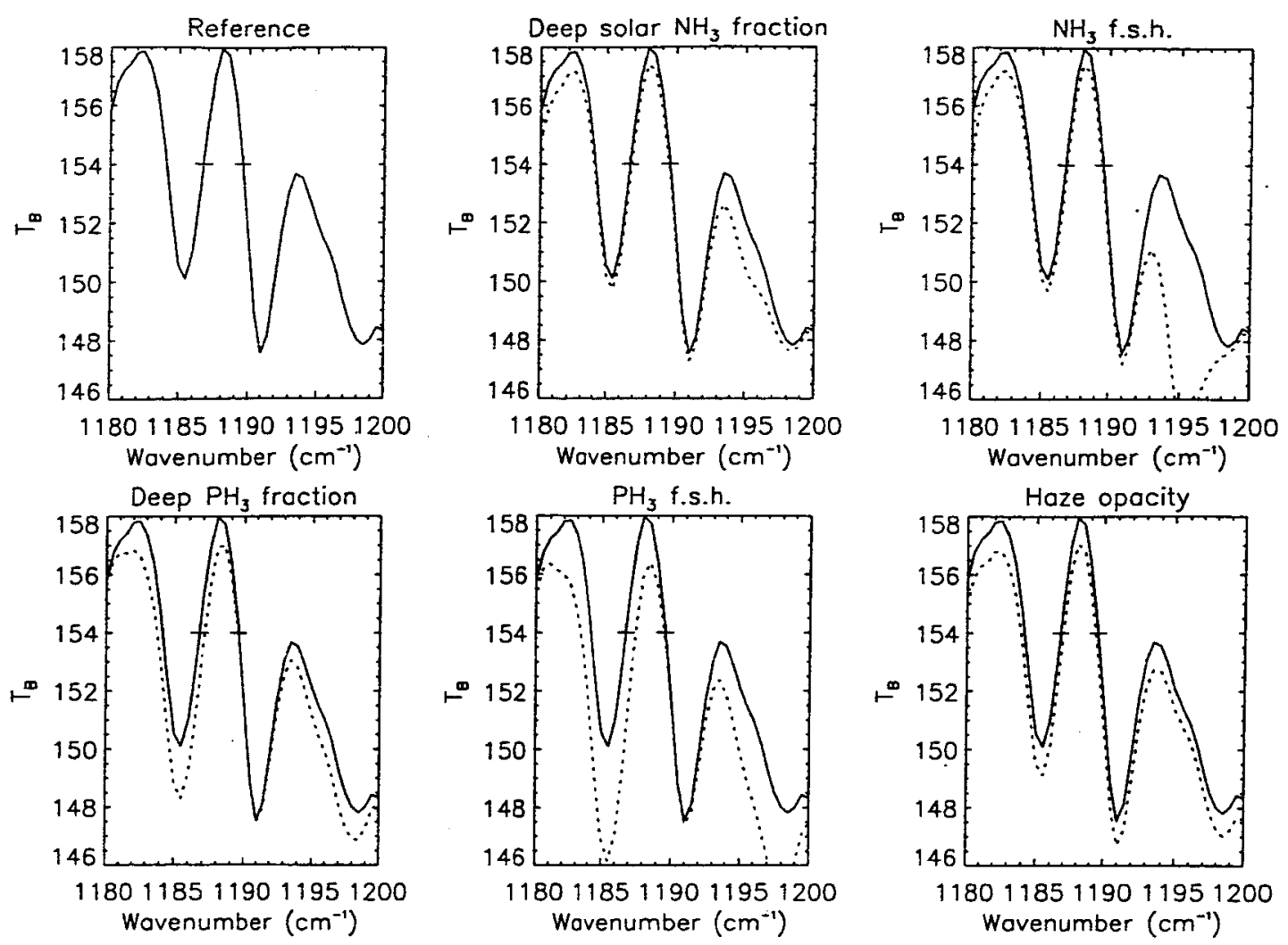

Figure 3. Sensitivity of synthetic spectra over a small wavelength range in the mid-IR to variations in the ammonia, phosphine and cloud parameters. The differential sensitivity of the $1186.7 \mathrm{~cm}^{-1}$ and $1190 \mathrm{~cm}^{-1}$ wavenumber pair to phosphine and equal sensitivity to the other parameters is very clear. 

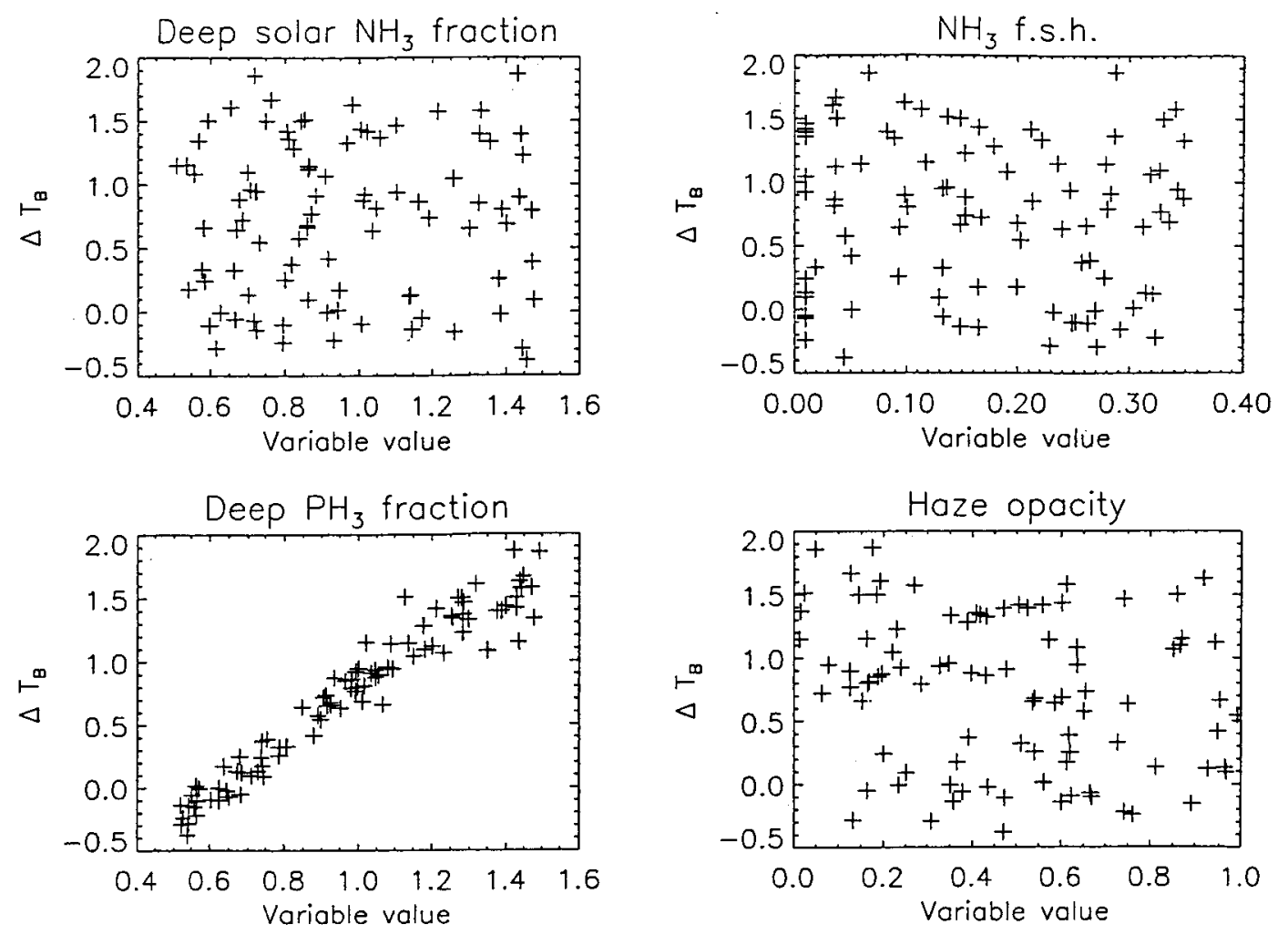

Figure 4. Variation of $\Delta T_{B}=T_{B}\left(1190 \mathrm{~cm}^{-1}\right)-T_{B}\left(1186.7 \mathrm{~cm}^{-1}\right)$ with parameters used to generate the suite of 92 synthetic spectra described in the text. The correlation with the phosphine abundances is very clear, as is the lack of correlation with other parameters. 

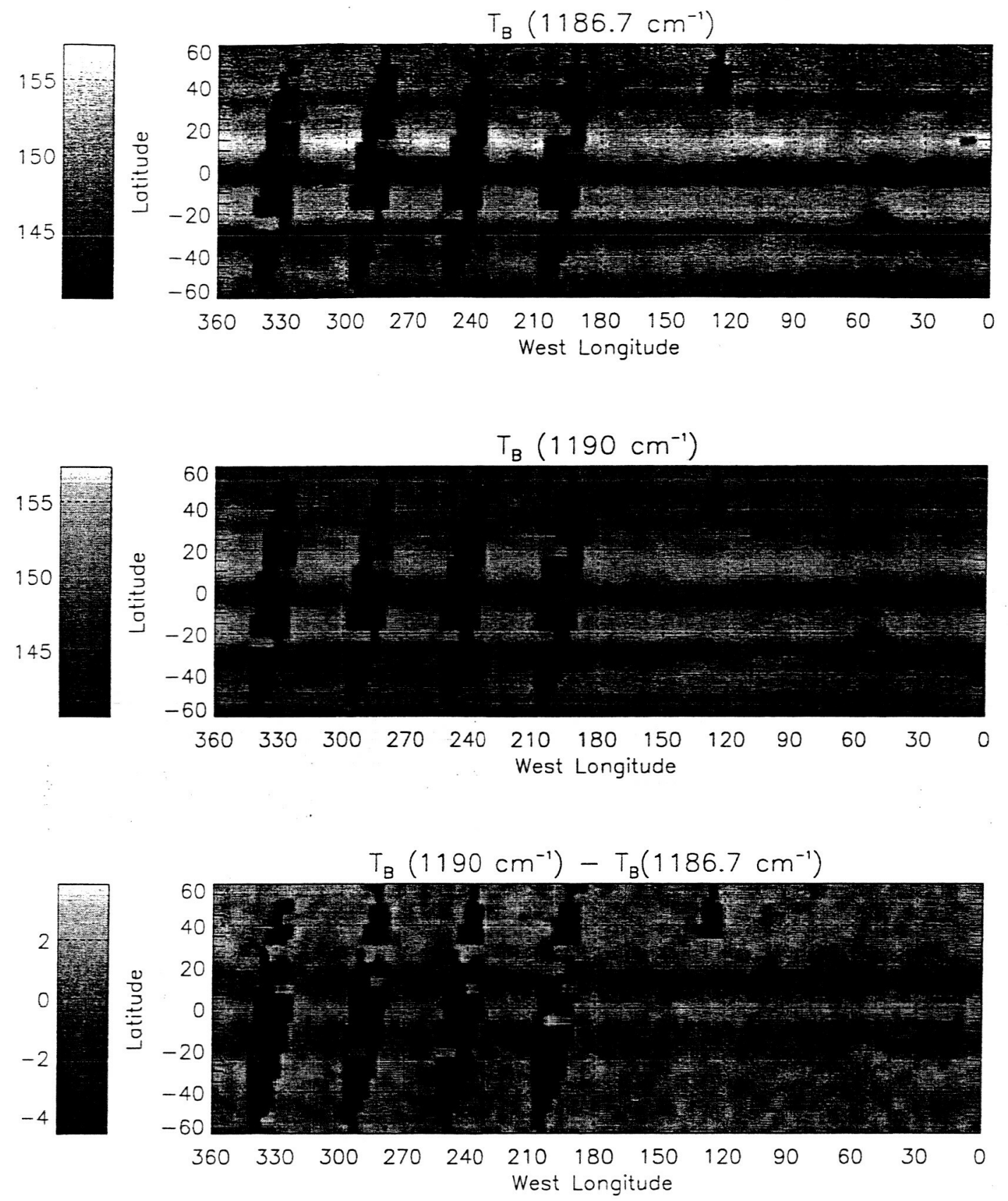

Figure 5. Brightness temperature maps at 1186.7 and $1190 \mathrm{~cm}^{-1}$ between $60^{\circ} \mathrm{S}$ and $60^{\circ} \mathrm{N}$ for the ATMOS02A observation, together with the variation of $\Delta T_{B}=T_{B}$ $\left(1190 \mathrm{~cm}^{-1}\right)-T_{B}\left(1186.7 \mathrm{~cm}^{-1}\right)$. Positive $\Delta T_{B}$ values indicate enhancement of phosphine. The dark, near-vertical stripes are missing data. The $\Delta T_{B}$ signal appears enhanced in the EZ, and depleted in the SEB and NEB. A possible enhancement at the northern edge of the GRS $20^{\circ} \mathrm{S}, 55^{\circ} \mathrm{W}$ can also just be seen. 

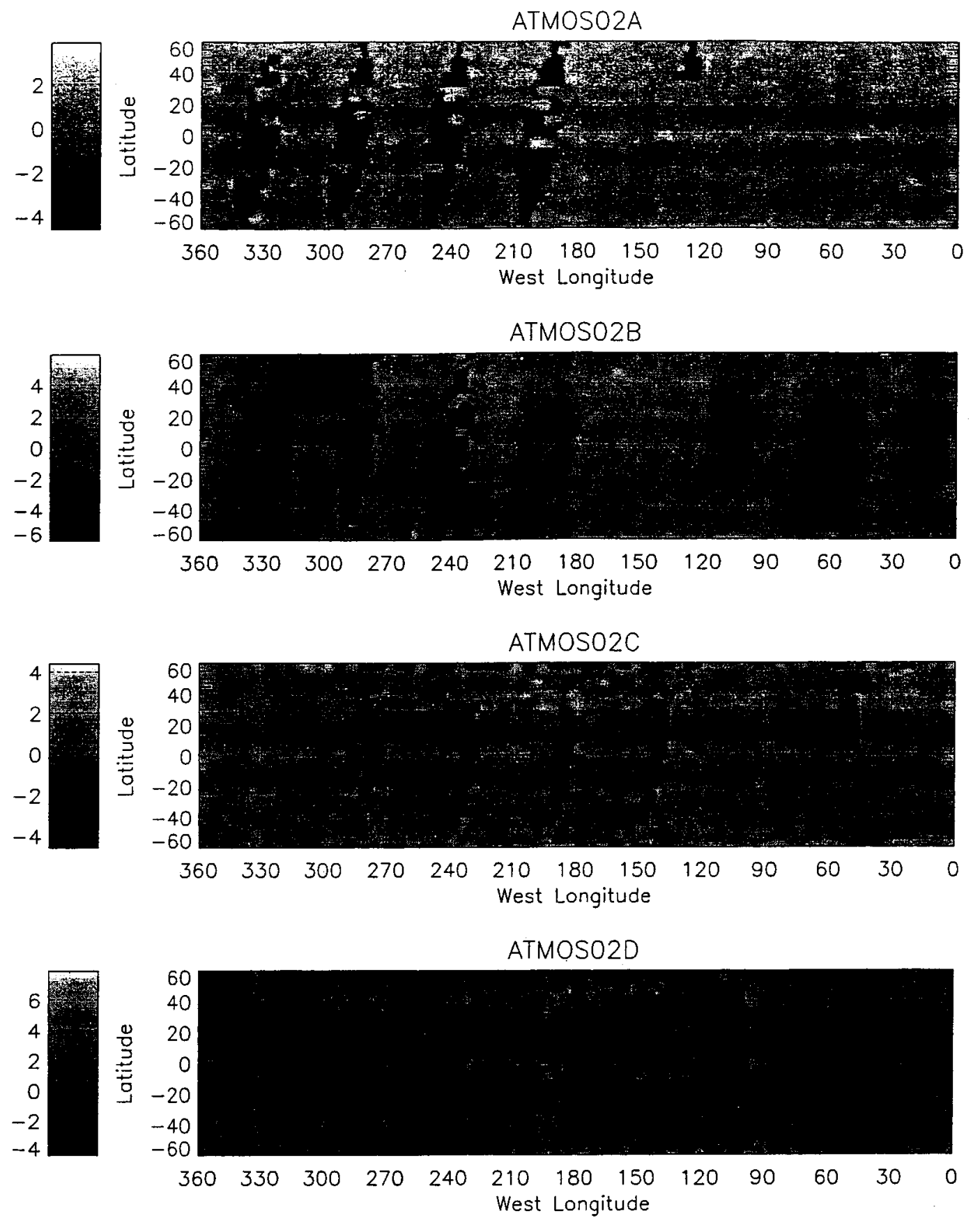

Figure 6. Maps of $\Delta T_{B}$ (proportional to phosphine enhancement) for all four of the ATMOS02A datasets. All four maps can be seen to have missing data but the latitudinal variation of the $\Delta T_{B}$ signal is clear in all, as is the slight enhancement at the northern edge of the GRS. The banding in the ATMOS02B map is due to a calibration shift in this dataset which occured half-way during the observation, and which is yet to be corrected for. 


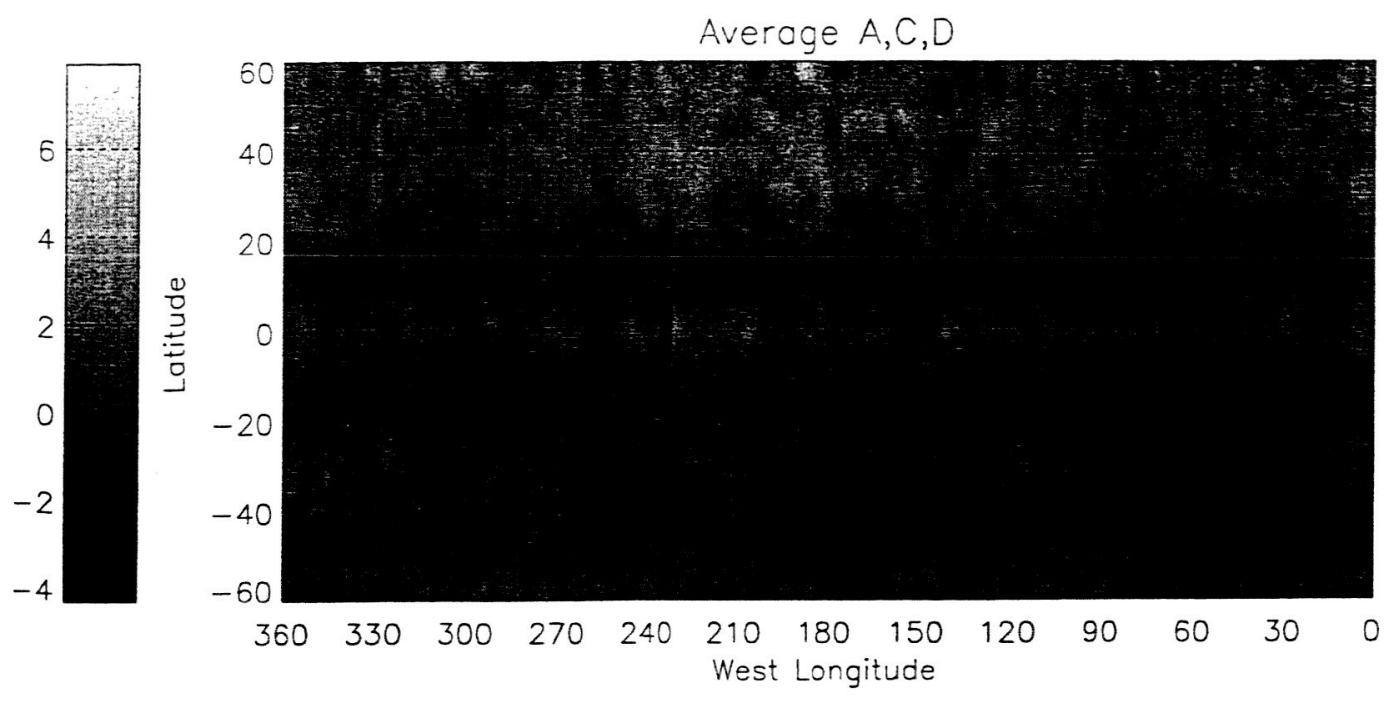

Figure 7. Average of $\Delta T_{B}$ (or phosphine enhancement) for ATMOS02 datasets $A, C$ and D. The ATMOS02B map was neglected due to the calibration uncertainties. The latitudinal variation in $\Delta T_{B}$ is clear, as is the increased signal to the northern edge of the GRS. 

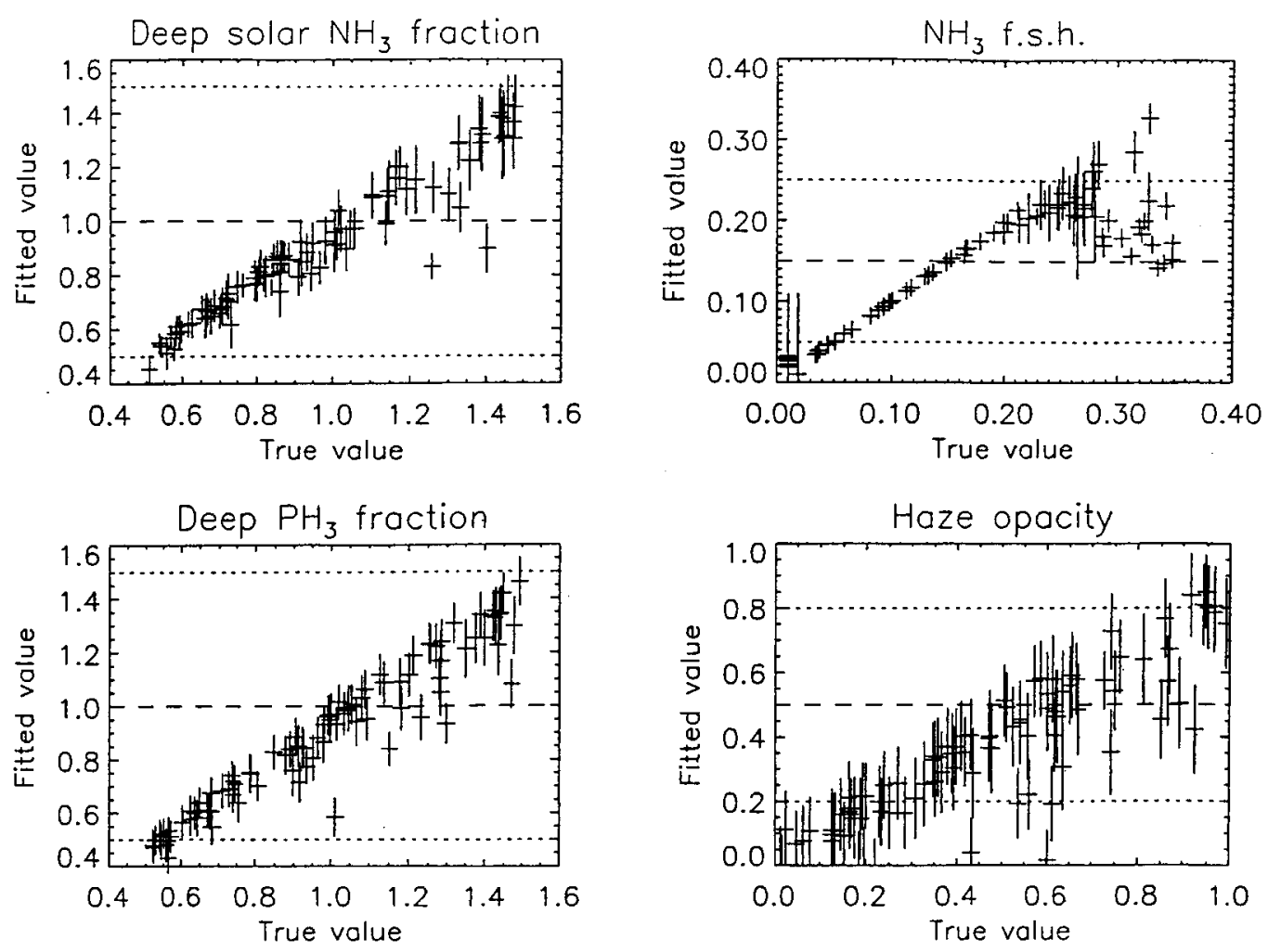

Figure 8. Validation of retrieval algorithm for set of synthetic test spectra. The four plots show the results for, respectively, deep $\mathrm{NH}_{3}$ solar fraction (i.e. relative to a v.m.r. of $2.19 \times 10^{-4}$ ), $\mathrm{NH}_{3}$ fractional scale height (f.s.h.), deep $\mathrm{PH}_{3}$ fraction (relative to the reference value of $6 \times 10^{-7}$ ) and cloud (or haze) opacity. The 'true' value of the parameter used to generate the spectrum is plotted along the $\mathrm{x}$-axis, while the fitted value and error is plotted on the y-axis. The a priori estimates of the retrieved parameters are indicated by the dashed lines, and the $a$ priori error limits by the dotted lines. The agreement between true and retrieved parameters is mostly very good. The peculiar behaviour of the ammonia fractional scale height results for true values greater than 0.25 is due to ammonia saturating in the model used to generate the synthetic spectra leading to lower than intended abundances of ammonia at higher altitudes. 

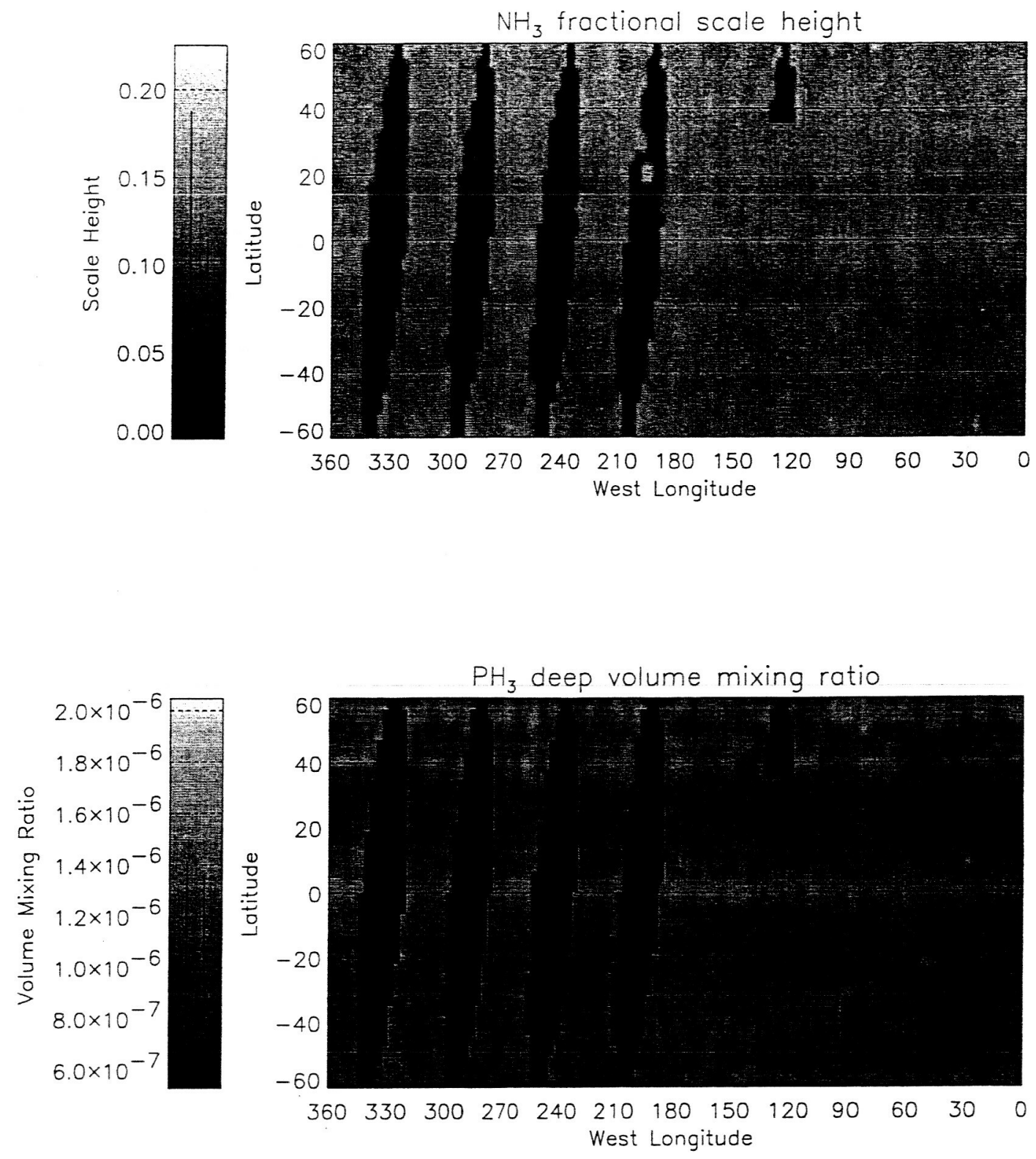

Figure 9. Retrievals of the ammonia fractional scale height and deep phosphine volume mixing ratio between $60^{\circ} \mathrm{S}$ and $60^{\circ} \mathrm{N}$ for the ATMOS02A map using the optimal estimation retrieval model. The averaged retrieved values are indicated by the horizontal lines in the scale bars and the individual a priori and retrieved error ranges are indicated by the two vertical bars respectively for each map. Both parameters show similar meridional variation with increased values over the EZ and decreased 
values over the NEB and SEB. However at mid-latitudes, although the ammonia f.s.h. distribution shows no North/South asymmetry, the phosphine distribution appears to be enhanced in the northern hemisphere. The possible enhancement of phosphine to the northern edge of the GRS is also apparent, as is the reduction of the ammonia f.s.h. over the centre of the spot.

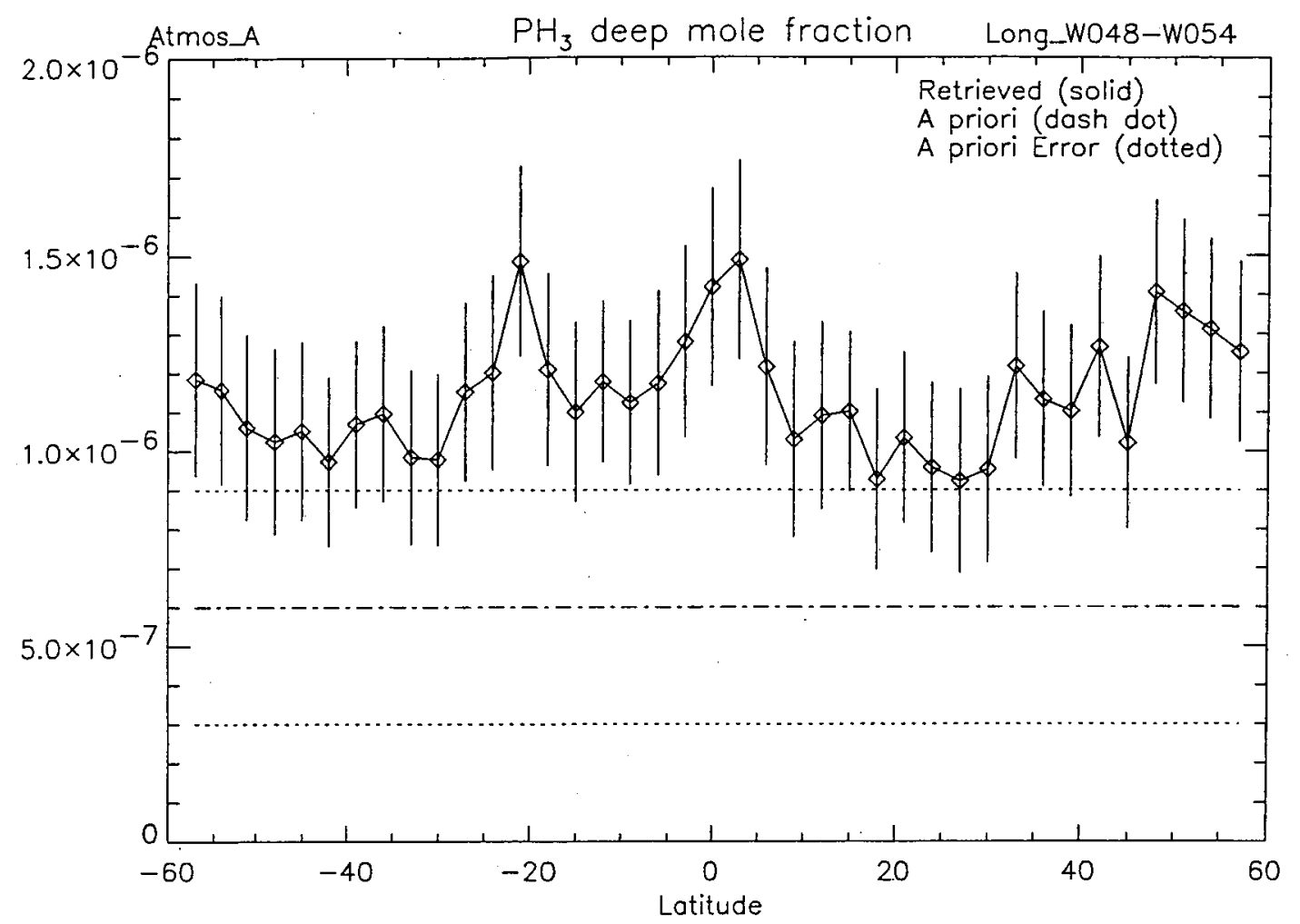

Figure 10. Variation of deep phosphine mole fraction (or v.m.r.) with latitude in the longitude strip passing through the GRS. The meridional variation of phosphine is clear as is the apparent enhancement over the GRS at $21^{\circ} \mathrm{S}$. In this plot the dash-dot line is the a priori phosphine abundance and the dotted lines indicate the a priori error limits. The retrieved phosphine values are shown by the solid line with the vertical bars indicating the retrieval error. 


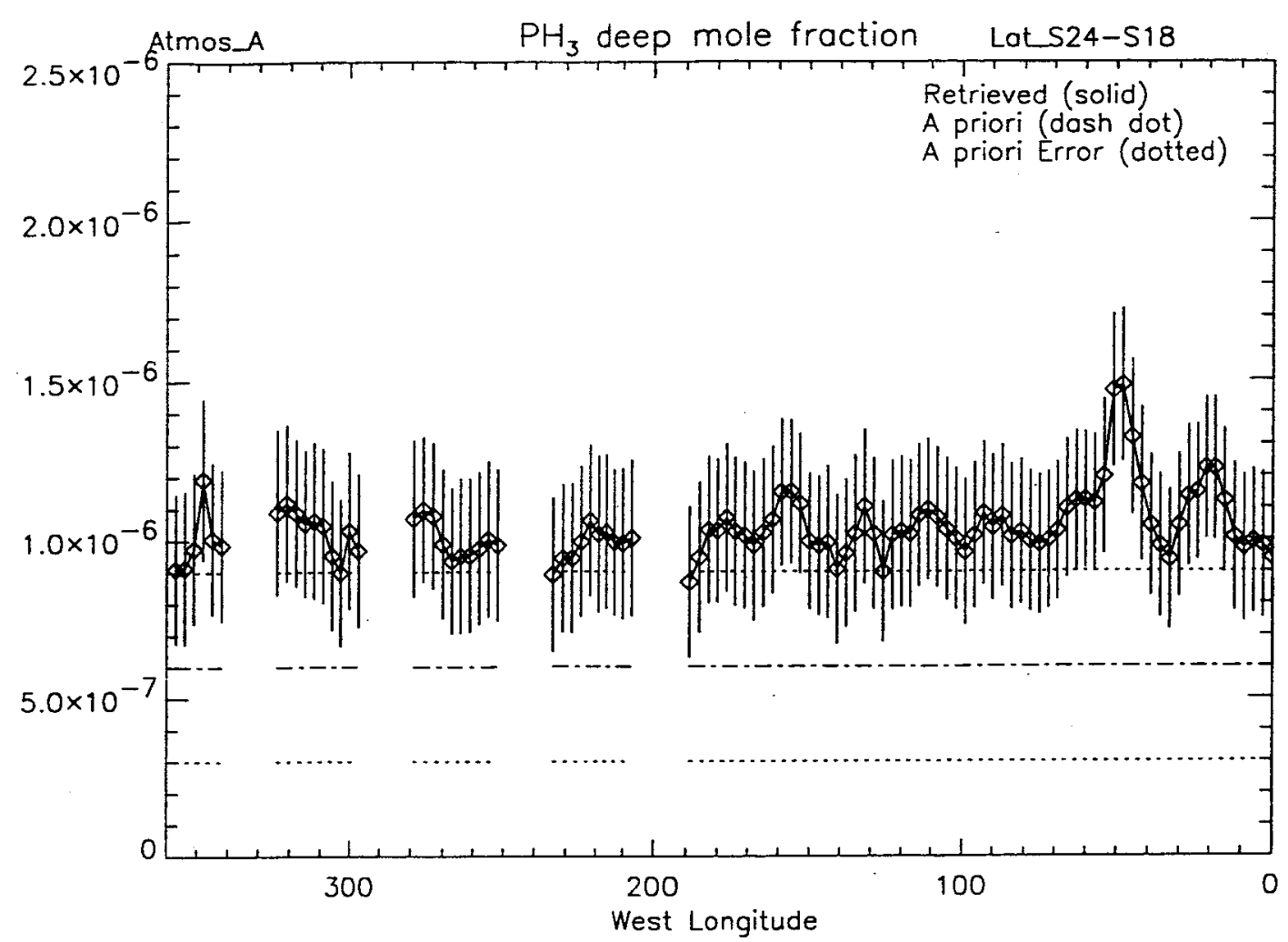

Figure 11. Variation of deep phosphine v.m.r. with longitude in the latitude strip passing through the GRS. The identification of the lines is identical to those in Figure 10. The apparent enhancement of phosphine over the GRS at $55^{\circ} \mathrm{W}$ is clear. 